\title{
Recent developments in neuropsychological endophenotypes for schizophrenia: Development of the MATRICS battery, liability syndromes and the near future
}

\author{
STONE William $\mathrm{S}^{1^{*}} \&$ HSI Xiaolu ${ }^{1,2}$ \\ ${ }^{1}$ Department of Psychiatry, Massachusetts Mental Health Center Division of Beth Israel Deaconess Medical Center, Harvard Medical School, \\ Boston, MA 02115-6092, USA; \\ ${ }^{2}$ MIT Medical, Massachusetts Institute of Technology (MIT), Cambridge, MA 02139, USA
}

Received April 3, 2011; accepted June 15, 2011; published online September 12, 2011

\begin{abstract}
Cognitive deficits are now recognized widely as core features of schizophrenia, and as major contributors to the clinical outcome of the disorder. They are also studied widely as 'endophenotypes', reflecting a growing consensus that schizophrenia is a broader, more multidimensional illness than the diagnostic criteria required for its formal diagnosis. This evolving view of cognition underlies its utilization in recent initiatives for intervention and assessment in schizophrenia. Two of these initiatives are reviewed in this paper. The first focuses on the development and validation of the MATRICS Cognitive Consensus Battery, a standardized battery of neuropsychological tests developed to assess the effectiveness of cognitive enhancing treatments in schizophrenia. A part of this effort includes the identification of performance-based, 'co-primary' measures of functional capacity that are related to cognition, and that are likely to show improvement at least partly as a function of improved cognition. The second initiative involves efforts to utilize neuropsychological deficits in the identification, validation and remediation of a liability syndrome for schizophrenia ('schizotaxia'). The discussion of this effort focuses on the development of a syndrome that is both measurable and meaningful clinically, and that may provide useful intervention targets. The utilization of cognition in both of these initiatives underscores its functional importance in the clinical outcome of schizophrenia. Moreover, it helps to illuminate indicators of liability for schizophrenia that might be amenable to remediation.
\end{abstract}

schizophrenia, schizotaxia, cognition, liability syndrome, MATRICS, neuropsychology

Citation: Stone W S, Hsi X. Recent developments in neuropsychological endophenotypes for schizophrenia: Development of the MATRICS battery, liability syndromes and the near future. Chinese Sci Bull, 2011, 56: 3385-3393, doi: 10.1007/s11434-011-4759-6

Although genetic contributions to schizophrenia are both well-accepted and compelling [1-4], the identity of most genes that increase susceptibility to the disorder - and the biological mechanisms by which they act - are largely unknown. One approach to understand how genetic determinants of schizophrenia leads to the disorder capitalizes on the idea that schizophrenia is broader than the DSM-IV or ICD-10 syndromes that are required for its diagnosis [5]. The importance of identifying specific abnormalities (i.e. 'endophenotypes') that reflect social, psychophysiological, neuropsychological, biological and other dimensions of

*Corresponding author (email: wstone@bidmc.harvard.edu) dysfunction reflects a growing awareness that multidimensional expressions of psychiatric disorders can advance the search for underlying etiological or modulatory factors [3,6-11]. Moreover, specific endophenotypes may be more amenable to objective measurement than symptoms, presumably reflecting variation among smaller numbers of genes than do more distal clinical symptoms $[6,12,13]$, and are heritable $[14,15]$. These points all support the usefulness of endophenotypes in genetic studies [5,6,12,13,16-21].

Among putative endophenotypes, examinations of neuropsychological functions have shown some of the most robust deficits, especially in domains such as overall cognitive ability (e.g. IQ), executive functions, sustained attention/ 
vigilance and verbal declarative memory [22-25]. In addition to their potential roles in furthering an understanding of the genetic etiology of schizophrenia, neuropsychological endophenotypes are significant for clinical/functional reasons. One of the most important of these are the effects of cognition on clinical and functional outcomes [26,27], such as the abilities to function in the community and to learn social skills. Notably, neuropsychological function are often unrelated to clinical symptom severity [28], and thus reflect different dimensions of function, at least in part. The magnitude, breadth and functional significance of these dimensions have important implications for the utilization of neuropsychological endophenotypes, both in treatment and assessment. This paper examines two of these implications. The first involves current attempts to develop a standardized battery of neuropsychological tests for use in clinical trials of cognitive enhancement. The second involves the use of putative neuropsychological endophenotypes in the development of a liability syndrome to identify individuals at risk for developing schizophrenia, and to identify treatment targets to attenuate or even prevent the development of the disorder.

\section{Development of a standardized cognitive battery for schizophrenia studies construction of the MATRICS cognitive consensus battery}

The need to compare findings across studies gives rise to the need to develop a standardized battery of neuropsychological tests to use in clinical trials of cognitively enhancing treatments in schizophrenia. It especially reflects the difficulties in interpretation that results from comparing different tests, test orders and administration times, among other protocol differences between studies. This need was addressed in the (American) National Institute of Mental Health (NIMH) initiative to encourage the development of novel interventions to attenuate cognitive deficits in schizophrenia, called Measurement and Treatment Research to Improve Cognition in Schizophrenia (MATRICS), which included the development of a reliable, valid cognitive consensus battery to use in clinical trials.

The development of the MATRICS battery to this point has been well documented [29,30], and will be summarized briefly, in the context of salient issues concerning the nature and assessment of cognitive deficits in schizophrenia. Initial decisions about the nature and breadth of the battery were determined by a survey of 68 experts [31]. Significant issues in this process were both practical and conceptual in nature. Practical concerns, for example, included the length of the battery, which led to a consensus that it should not exceed $90 \mathrm{~min}$. Moreover, each test in the battery should not exceed $15 \mathrm{~min}$, and should have good reliability and validity. The availability of comparable alternate forms for individual tests was important. Each test in the battery - and the bat- tery itself - should be feasible for use in clinical trials of pharmacological agents with proposed cognition enhancing properties. To address these concerns, the battery needed to demonstrate good test-retest reliability, lend itself to repeated measurements, demonstrate acceptable degrees of tolerance for patients and show adequate sensitivity to change upon retesting, as a result of pharmacological interventions.

Important conceptual issues were also considered, including the type of tests to include in the battery. This issue is complicated for a number of reasons, including the broad range of cognitive deficits in schizophrenia. This range can be conceptualized in at least two ways. First, breadth of deficits can be considered to reflect a single generalized deficit, which might be reflected by measures that assess generalized functioning, such as Full Scale IQ scores derived from Wechsler intelligence tests. Impairments in broad measures of cognition, however, also occur in the context of broad neuropsychological impairments across multiple domains. This raises the question of whether separable cognitive deficits really occur in schizophrenia, or whether they reflect a more generalized or widespread deficit that is evident in whichever cognitive domain is assessed. Several studies present evidence for broad general factors [28]. Dickinson et al. [32] showed that $47 \%$ of the variance in a battery of cognitive tests was attributable to a schizophrenia diagnosis, with $65 \%$ of this diagnosis-related finding attributable to a single, common factor. A subsequent study showed that $63.6 \%$ of the diagnosis-related variance was attributable to a common factor, with much smaller percentages attributable to performance in other cognitive domains, such as verbal memory (13.8\%) and processing speed $(9.1 \%)$ [33]. The same group demonstrated that, compared to healthy controls, schizophrenia patients showed higher correlations between composite scores of neuropsychological functions in a study of confirmatory factor analysis, consistent with the hypothesis that patients demonstrated a more generalized cognitive ability (or deficit) than controls [34].

Nevertheless, these and other studies show that the choice between general and more specific cognitive abilities is not necessarily a mutually exclusive one, as both general and more specific cognitive deficits in schizophrenia occur simultaneously $[32,35,36]$. As importantly, the presence of one or more common cognitive factors does not mean that eventual treatments would affect all dimensions of cognition equally. Neuropsychological test performance in healthy control samples is often variable $[37,38]$ and subject to dissociation (e.g., the patient H.M. developed profound temporal lobe amnesia following surgery for epilepsy, but retained intact immediate memory and above-average overall cognitive abilities [39]). Similarly, cognitive dysfunction is also likely to be dissociable, with potential treatments for schizophrenia influencing some dimensions of cognition more effectively than others. 
As part of the effort to develop the MATRICS battery, evidence for separable cognitive factors was evaluated empirically, with an emphasis on factor analytic studies [36]. This review yielded 7 distinct, replicated dimensions of cognitive dysfunction in schizophrenia, including: Speed of Processing, Attention/Vigilance, Working Memory, Verbal Learning and Memory, Visual Learning and Memory, Reasoning and Problem Solving, and Verbal Comprehension. Verbal Comprehension was considered inappropriate for inclusion in the battery because it was considered less likely than the other dimensions to change with treatment. Social Cognition was added to the list because of growing interest in this particular dimension of function, and because of a more general interest in understanding how changes in cognition affect other functional domains. These seven domains were recommended for inclusion in the MATRICS-NIMH consensus cognitive battery (MCCB).

Consideration of conceptual, psychometric and pragmatic factors produced an initial candidate list of 90 tests, which were reduced to 36 tests in the seven cognitive dimensions described above [29]. These tests were then evaluated using the RAND/UCLA appropriateness method, which involves a series of steps that are applied iteratively to increase rater agreement among a panel of experts [40]. The panel consisted of experts in several relevant fields who rated the each test according to 5 criteria. These ratings were used to create a beta version of the battery that included 20 tests that could be classified in 14 cognitive domains. The MATRICS Psychometric and Standardization Study (PASS) was then conducted on a sample of patients with schizophrenia who were assessed at baseline and then again after 4 weeks, to develop a final version of the battery. Selection of tests for the final version was based on the psychometric properties of the tests in the beta version (e.g., test-retest reliability, and utility as a repeated measure), their practicality, and their tolerability. Following a discussion of each candidate test, experts on the MATRICS Neurocognition Committee rated them to select 10 tests for the final battery, which is shown in Table 1 along with the cognitive domains they represent. These cognitive domains were among those that were determined to be separable earlier [36]. Once the tests were selected, they were brought together into a single battery [41], with a website to facilitate acquisition and use of the battery (http: //www.matricsinc.org).

\section{MATRICS co-norming and validation}

Once the individual tests were selected for the MATRICS battery, the psychometric properties of the battery itself were established. The basis of these studies involved the need to establish normative performance on the tests in the same battery, using the same normative source (i.e. co-norming). This step was essential because performance on individual tests in the MATRICS battery were normed originally on different samples with different characteristics [30]. In the absence of co-norming, performance on these tests cannot be compared with each other in a straightforward manner. Moreover, base rates of differences in performance on different tests, expected levels of variability and even normative performance would be difficulty to establish, as would the development of composite scores or an overall unitary measure of performance. Consequently, performance on the MATRICS battery considered as a whole was compared in patients with schizophrenia to the performance of individuals recruited from the community, for the purpose of establishing normative levels of performance [30]. The community sample was recruited to reflect representative geographic regions of the United States, and also representative distributions of age, gender, education and ethnicity. The data from this study was used to develop the MATRICS computer scoring program, and for options to include or not include demographic covariates (i.e. no covariates, age and gender covariates, or age, gender and education covariates). The results confirmed the prominent cognitive deficits in schizophrenia patients, and also showed consistently significant effects of age and education on per-

Table 1 MATRICS cognitive consensus battery (MCCB) $)^{\text {a) }}$

\begin{tabular}{|c|c|}
\hline Test & Cognitive domain \\
\hline 1. Trail Making Test, Part A & Speed of Processing \\
\hline 2. Brief Assessment of Cognition in Schizophrenia (BACS): Symbol-Coding & Speed of Processing \\
\hline 4. Wechsler Memory Scale — III (WMS-III): Spatial Span & Working Memory (nonverbal) \\
\hline 5. Letter-Number Span & Working Memory (verbal) \\
\hline 6. Neuropsychological Assessment Battery (NAB): Mazes & Reasoning and Problem Solving \\
\hline 7. Brief Visual Spatial Memory Test — Revised & Visual Learning \\
\hline 8. Category Fluency (Animal Naming) & Speed of Processing \\
\hline 9. Mayer-Salovey-Caruso Emotional Intelligence Test (MSCEIT): Managing Emotions & Social Cognition \\
\hline 10. Continuous Performance Test — Identical Pairs & Attention/Vigilance \\
\hline
\end{tabular}

a) Tests are listed in order of administration. Estimated administration time $=63.5 \mathrm{~min}$. 
formance. Effects of gender varied more between individual tests. A recent follow-up study of this sample showed that the speed of processing and the social cognition domains best distinguished individuals with schizophrenia from the community sample [42]. The speed of processing, visual learning and attention/vigilance domains best distinguished individuals with schizophrenia who were competitively (i.e. gainfully) employed from those who were not.

Validation studies involving the MATRICS battery have been encouraging. For example, the battery had been translated into 11 languages as of 2010 [43], and is being translated into more languages in 2011 for use in international trials. A recent, 29-site trial of the effects of two antipsychotic medications administered to stable outpatients with schizophrenia in the United States confirmed that the MATRICS battery was sensitive to cognitive deficits, had good test-retest reliability and showed small practice effects [44].

Another important initiative involves the development of 'co-primary' measures to accompany the MATRICS battery. This effort derives from a position adopted by The U.S. Food and Drug Administration (FDA) that improvement on neuropsychological tests such as those in the MATRICS battery is a necessary, but not a sufficient condition for approval of a drug for cognitive enhancement in schizophrenia [45]. This position reflects the view that performance on cognitive measures is not related clearly enough to performance of 'real-world' tasks. Consequently, the FDA required that pharmacological treatments for cognition must also improve performance on a functionally meaningful 'co-primary' measure that has more face validity for consumers and clinicians than neuropsychological tests.

Methods for evaluating performance-based and interview-based measures were developed as part of the PASS study [46]. The Validation of Intermediate Measures study (VIM) then utilized and extended these methods to determine the psychometric properties, utility and practicality of several potential co-primary measures [47]. One hundred and sixty-six patients with schizophrenia were assessed at baseline and 144 were retested 4 weeks later. Subjects received clinical assessments, the MATRICS battery, and performance-based (the Independent Living Scales, the Test of Adaptive Behavior in Schizophrenia, and the UCSD Performance-based Skills Assessment) and interview-based (the Cognitive Assessment Interview and the Clinical Global Impression for Cognition) candidate co-primary measures. The performance-based measures had higher correlations with cognitive function than did the interview-based measures. Overall, the UCSD Performance-based Skills Assessment had the strongest overall properties, and the highest correlation with performance on the MATRICS battery (0.67). Full versions of the performance-based tests had better psychometric properties than short versions. Keefe and colleagues also showed a similar correlation between MATRICS performance and a brief version of the
UCSD Performance-Based Skills Assessment [44].

In a relatively short period, the MATRICS battery has become a standard measure for the assessment of cognition in schizophrenia, and particularly for the assessment of pharmacological treatments aimed at improving cognition in schizophrenia. Nevertheless, the extent of its utility and usefulness still requires additional validation. The likelihood that MATRICS performance measures will serve as neuropsychological endophenotypes that might be useful for genetic studies, for example, is largely unexplored. It is also premature to conclude at this point when the MATRICS battery will be more or less useful than other cognitive batteries in clinical trials of cognition enhancing drugs [48,49].

The effort that went into its construction and validation, however, underscores a growing recognition of the functional importance of cognition in schizophrenia outcomes, and of the possibility that cognitive deficits/endophenotypes could be viable treatment targets in the near future. Another critical area that involves neuropsychological endophenotypes in particular and neuropsychological function in general, is in the identification of liability for schizophrenia. One conceptualization of this liability, and its assessment, is considered next.

\section{Liability syndromes for schizophrenia}

\subsection{Foundations of the concept}

The notion of liability to schizophrenia is not new. Bleuler [50] was among the first of many clinicians and researchers to note that individuals who develop schizophrenia often show abnormalities (e.g. interpersonal oddness or otherwise impaired social function) as children or adolescents, well before they develop schizophrenia. Family, twin and adoption studies have long demonstrated that close biological relatives of individuals with schizophrenia show greater risks for developing schizophrenia or related disorders than the general population $[1,51,52]$. Evidence that a significant portion of this liability reflects a genetic etiology is compelling at this point $[1-4,53,54]$, and contributes to the conceptualization of endophenotypes as heritable abnormalities that are associated with the disorder and that occur at elevated rates in 'unaffected' relatives who share many genes with their ill relatives [6]. In this context, unusual behaviors in non-psychotic relatives of patients with schizophrenia were also observed frequently. Rado noted that "schizotypes" and "schizotypal behaviors" resulted from a genetic liability to schizophrenia $[55,56]$, which was integrated into a model proposed by Meehl [57,58] in which a major gene (a "schizogene") produced a "neural integrative defect" that he referred to as "schizotaxia". The underlying neural defect produced an observable phenotype called "schizotypy", which is a type of personality organization that interacted with environmental variables such as social learning and other, polygenic factors (e.g., a predisposition to high or 
low anxiety, or to different types of temperament). When the environment and other genetic circumstances were favorable in individuals who carried the schizogene, the observable phenotype would consist of only minor clinical symptoms (e.g., 'compensated schizotypy'), but when other genetic and/or environmental circumstances were less favorable, then more severe, decompensated conditions would result, including schizophrenia.

Over the next 3-4 decades, schizotaxia was used as a general term to describe the premorbid, neurological liability for, and basis of, schizophrenia. In part because schizotaxia was unobservable directly in Meehl's conceptualization, it was not defined operationally or studied as a clinical syndrome. In contrast, Meehl's conception of the most direct consequence of schizotaxia - schizotypy - was considered observable, and that concept did enter the psychiatric nosology in the modified form of 'schizotypal personality disorder'. By the 1990s, however, a growing body of literature showed that a subset of non-psychotic relatives of people with schizophrenia showed a broad range of measurable abnormalities in brain structure and function, neurophysiological aspects of information processing such as eye tracking and various sensory gating paradigms, neuropsychological function, and social functioning, among others $[7,8,17,59]$. These findings supported the view that the notion of liability might be amenable to operational definitions, whereas it had not been previously.

Other factors contributed to the development of this idea. The focus on non-psychotic relatives, who could be studied without the confounding effects of psychosis, medication or chronic illness, produced a paradigm shift in the field [60], away from formal diagnostic clinical symptoms in patients, and towards biological, neuropsychological, social and other markers of schizophrenia disease. This shift focused renewed attention on endophenotypes, which helped to provide a conceptual understanding of the schizophreniarelated abnormalities that were identified in relatives [13]. As the focus shifted away from diagnostic criteria for schizophrenia, it also shifted away (somewhat) from psychosis, and towards problems (e.g., neuropsychological deficits and social functioning deficits) that onset earlier than psychosis that might reflect the liability to develop schizophrenia. Longitudinal findings from genetic high risk studies showing neuropsychological, social/interpersonal and motor and abnormalities in the children of parents with schizophrenia that were associated with the development of subsequent psychosis, for example, were major contributors to this research $[61,62]$. In the same period, replications of genetic linkage studies gave new hope that eventually, susceptibility genes for schizophrenia might be identified, and would contribute to the early identification of individuals most at risk for developing schizophrenia [52]. Early identification, in turn, would facilitate early intervention efforts, and hopefully, at some point, prevention.

Each of these lines of research promoted a broader view of schizophrenia than the DSM or ICD symptoms required to diagnose it. No strong, single predictor of schizophrenia emerged from this research, however, to predict the development of the disorder reliably [63]. Many abnormalities that show predictive power when studied retrospectively are nonspecific, and are likely to contribute to elevated false positive rates and lower levels of specificity [20]. Moreover, even subjects who demonstrate positive symptoms (as demonstrated by elevated Scale of Prodromal Symptoms (SOPS) scores, for example) may remit or otherwise do not progress to develop schizophrenia [64-66]. A more accurate approach may involve the use of multiple measures or combinations of measures $[67,68]$, but the prediction of schizophrenia, particularly for individuals, remains at an early stage of development.

\subsection{Development and validation}

In this broad context, we reformulated the term schizotaxia in the 1990s to integrate newer data [59]. Our conception of schizotaxia was consistent with Meehl's view of it as the underlying liability among people predisposed to schizophrenia, though aspects of his view were modified. Perhaps most importantly among these differences, we proposed a provisional, operational definition of schizotaxia [69] that allows the concept to be validated or disconfirmed experimentally. At this point, the proposed syndrome applies to adult, non-psychotic, biological, first-degree relatives of individuals with schizophrenia. The initial research criteria involved a subset of theoretically important symptoms/ deficits that were relatively well-studied at the time, including negative symptoms and specific deficits in neuropsychological functioning. Negative symptoms were included because they (flat affect and avolition especially) are often elevated in families with a schizophrenic member, in contrast to positive symptoms [e.g., 70]. In this view, negative symptoms in schizotaxia are essentially the same as negative symptoms in DSM-IV schizotypal personality disorder. In this sense, they represent a 'negative schizotypy' dimension, without the positive (or disorganized) dimension that together constitutes the DSM-IV disorder [71]. In our pilot studies, anhedonia (e.g., few interests or sources of pleasure) and asociality (e.g., few romantic relationships or close friends) were among the most common negative symptoms observed in individuals with schizotaxia [69].

Our initial criteria for schizotaxia also included neuropsychological deficits in verbal memory, attention and/or executive functions. Deficits in these neuropsychological domains are core features of schizophrenia that also occur frequently in relatives [7,23,72-75]. In addition to negative symptoms and cognitive deficits, several inclusion and exclusion criteria were intended to minimize false positive diagnoses (e.g., negative symptoms or cognitive problems due to known neurological or medical conditions, current substance dependence, history of electroconvulsive treat- 
ment, or English spoken as a second language), and subjects with any lifetime history of a psychotic disorder [69]. Using these provisional, conceptually-based criteria, we assessed a group of 27, non-psychotic, first degree relatives of individuals with schizophrenia [76]. Eight subjects met criteria for schizotaxia, and 19 did not. Comparisons between the two groups were made on several relatively independent, non-criterion clinical measures, including the Social Adjustment Scale (SAS) [77], the DSM-IV Global Adjustment Scale (GAS) [78], and the Symptom Checklist-90-Revised (SCL-90-R) [79], among others. The groups did not differ in age, education, paternal education, IQ or number of ill relatives. The comparisons demonstrated evidence of concurrent validity for the concept by showing that relatives who met criteria for schizotaxia also showed poorer functioning on these clinical measures compared to relatives who did not meet criteria, regardless of whether the measures were self-rated or rated (blindly) by the researchers.

As part of an attempt to determine whether symptoms of schizotaxia were amenable to intervention, we also administered a low dose of risperidone $(0.25$ to $2.0 \mathrm{mg})$ to 6 of the 8 subjects who met the schizotaxia criteria, and who agreed to a six-week, open-label course of the drug. Institutional oversight was provided by the Institutional Review Board (IRB) of the Massachusetts Mental Health Center (Boston, Massachusetts, USA). All subjects completed the protocol. Side effects were temporary and mild, and no abnormal movements were observed. Five of the 6 subjects showed improvement on a demanding test of auditory-verbal working memory, and showed reductions (25\% to $50 \%$ ) in the number of negative symptoms rated as at least moderate (i.e. scores of 3 or higher) on the Scale for Assessment of Negative Symptoms (SANS) [80].

The results of the pilot validation study and the response to the pharmacological probe were encouraging, and led to a larger investigation designed to replicate or modify the proposed liability syndrome in adult, non-psychotic, firstdegree biological relatives. Although the pilot study was conducted in Boston, the subsequent study was conducted at the Mental Health Institute, Second Xiangya Hospital of Central South University, Changsha, Hunan Province, People's Republic of China, and will be referred to as the 'Changsha Study' (Stone et al., submitted). The change in location reflected both the challenges involved in recruiting subjects locally who met our provisional criteria, and the opportunity to recruit appropriate subjects through collaborators who were working with larger numbers of schizophrenia families.

The clinical (negative symptoms) and neuropsychological domains used to assess schizotaxia were the same in the Changsha study as they were in the Boston pilot study. Individual neuropsychological tests, however, were changed, because most of the criterion tests used in Boston did not have normative values for Chinese samples. The criteria used in the pilot study with the measures we employed were stringent, but were effective in identifying almost a third of the first group of relatives we assessed (8/27) as meeting criteria for schizotaxia. These criteria identified a much lower percentage of relatives in the Chinese sample, using the modified battery of tests with Chinese norms. Consequently, we modified the criteria to make them less stringent, but we also performed cluster analyses to determine whether our two-group classification of relatives into schizotaxic and non-schizotaxic groups cohered empirically. In addition to the criterion measures, subjects also received additional measures of clinical, social, neuropsychological and medical function.

One hundred and eighty-nine relatives were assessed, along with 30 community comparison subjects (CCS). Using the modified, conceptually-based criteria, 86 subjects were classified as having schizotaxia (45.5\%), while 103 were not. In general, relatives classified as non-schizotaxic performed similar to the CCS group. Relatives classified as schizotaxic showed significantly lower/poorer performance on a variety of criterion-related and non-criterion-related neuropsychological, clinical and social measures, including the GAF and the SAS. Results of the cluster analysis confirmed the two-group solution, and confirmed the classification of most of the subjects. It did, however, reduce the number of subjects classified as schizotaxic from 86 to 53 $(28.0 \%)$, making it similar to the percentage we obtained in our pilot study in Boston, and it increased the non-schizotaxic relatives group to 135 . The cluster-derived groups showed the same pattern of relationships as the conceptually determined groups, but the effect sizes of several group differences increased, including the GAF and the SAS.

Subjects classified with schizotaxia were also recruited into a six-week intervention trial that involved administration of low doses of risperidone $(0.25$ to $2.0 \mathrm{mg}$ ) (Stone et al., submitted). Unlike our open-label pilot study, the trial utilized a double-blind, placebo-controlled design. Thirty-six of the 86 subjects who met criteria for schizotaxia agreed to enter the trial, of whom 20 were randomized to the risperidone group, and 16 were randomized to the placebo group. Side effects were mild and well tolerated generally, but one subject receiving risperidone did stop taking it $2 \mathrm{~d}$ before the completion of the protocol. The first set of data analyses focused on the criterion measures for schizotaxia, the noncriterion neuropsychological measures, and some of the non-criterion clinical measures, including the GAF and the SAS. The results showed significant improvement in the risperidone group in the criterion measures for executive function (i.e. fewer perseverative errors on the Wisconsin Card Sorting Test), and better self-rated social function on the SAS. The GAF scores did not change. The SANS scores did not change, either, which emphasizes the importance of follow-up initial findings obtained using open-label designs with more rigorous, double-blind protocols.

Overall, these results are encouraging, and emphasize the 
potential importance of cognition in the assessment of liability for schizophrenia. It should also be emphasized, however, that progress towards the identification of a liability syndrome for schizophrenia remains in the early stages of development. The use of different neuropsychological tests in the Boston and the Changsha samples, and the results of the cluster analysis, emphasize the provisional nature of the criteria at this point. The question of how to integrate other dimensions of function into a liability syndrome (e.g., structural and functional brain abnormalities, and electrophysiological deficits) remains an unexplored issue, as is the even greater fundamental question it raises about whether the liability for schizophrenia is best conceptualized as a single, large syndrome in which many symptoms may or may not appear in a particular individual, or whether there are really many liability syndromes that could be defined and validated. The results of the risperidone study also require caveats. Only some measures changed with treatment, which could have occurred by chance, and which will necessitate additional replication. Although our findings are potentially significant in showing that neuropsychological and other symptoms related to the liability for schizophrenia may be amenable to intervention, they do not provide sufficient justification for the clinical use of antipsychotic medications - and their potential medical and psychosocial side effects - in preventive roles at this time.

\section{Discussion and conclusions}

Schizophrenic illness is associated with numerous neuropsychological abnormalities that are not related formally to DSM-IV or ICD-10 diagnostic criteria for schizophrenia or for other disorders in the schizophrenia spectrum. Many of these abnormalities meet some or all criteria proposed for endophenotypes, and are core features of schizophrenic illness. The importance of these cognitive abnormalities is emphasized in the two types of initiatives discussed in this review. The first of them involves the development of the MATRICS battery for use in clinical trials of treatments that might affect cognition. The second involves efforts to identify the liability to schizophrenia. Both of these initiatives share features in common that reflect the evolution of thinking in the field. The most fundamental similarities include recognition of the importance of cognition in clinical outcome, and in measuring liability. In schizophrenia itself, cognition is a major determinant, for example, of whether individuals can perform activities of daily living, live independently, or work. In individuals at high risk for schizophrenia, cognition is an important predictor of whether they will develop psychosis. Moreover, these initiatives emphasize the functional importance of cognition in relation to clinical measures and to other measures of functional capacity.
The initiatives emphasized here also reflect another important development. Until recently, the possibility of ameliorating cognitive deficits in schizophrenia has seemed slight $[28,83,84]$. Both of the initiatives discussed here, however, reflect an evolving view that specific cognitive measures may serve as useful, viable treatment targets for efforts to remediate cognitive weaknesses in schizophrenia. These targets are evident once psychosis develops, but they may also be very important prior to the onset of psychosis.

Future research will be needed to continue to validate the utility of potential individual cognitive measures, as well as to validate the performance of batteries such as MATRICS and putative liability syndromes as a whole. These include their epidemiological properties such as base rates, their sensitivity to detect truly affected individuals, their specificity to reject truly unaffected individuals, and their sensitivity to change with intervention. Another critical area involves the need to understand further how cognition is related to other dimensions of schizophrenic illness, including both positive and negative symptoms, but also social, biological, genetic and other dimensions of function. The inclusion of cognition in broader consortium and other collaborative efforts to assess interrelationships across multiple dimensions of function will provide important catalysts for progress in each individual dimension. Hopefully, the growing emphasis on cognition in schizophrenia that is reflected by the recent initiatives discussed above will facilitate progress in both diagnosis and intervention in ways that will materially improve clinical outcomes.

This work was supported in part by Ortho-McNeil Janssen Scientific Affairs, LLC, and by National Institute of Mental Health Grant RO1MH065562 (COGS; Consortium on the Genetics of Schizophrenia).

1 Gottesman I I, Hanson D R. Human development: Biological and genetic processes. Annu Rev Psychol, 2005, 56: 263-286

2 Harrison P J, Weinberger D R. Schizophrenia genes, gene expression, and neuropathology: On the matter of their convergence. Mol Psychiatry, 2005, 10: 40-68

3 Allan C L, Cardno A G, McGuffin P. Schizophrenia: From genes to phenes to disease. Curr Psychiatry Rep, 2008, 10: 339-343

4 Craddock N, O'Donovan M C, Owen M J. Psychosis genetics: Modeling the relationship between schizophrenia, bipolar disorder and mixed (or "schizoaffective") psychoses. Schizophr Bull, 2009, 35: $482-490$

5 Tsuang M T, Stone W S, Faraone S V. Towards reformulating the diagnosis of schizophrenia. Am J Psychiatry, 2000, 147: 1041-1050

6 Gottesman I I, Gould T D. The endophenotype concept in psychiatry: Etymology and strategic intentions. Am J Psychiatry, 2003, 160, 636-645

7 Gur R E, Calkins M E, Gur R C, et al. The Consortium on the Genetics of Schizophrenia (COGS): Neurocognitive endophenotypes. Schizophr Bull, 2007, 33: 49-68

8 Turetsky B I, Calkins M E, Light G A, et al. Neurophysiological endophenotypes of schizophrenia: The viability of selected candidate measures. Schizophr Bull, 2007, 33: 69-94

9 Chan R C, Gottesman I I. Neurological soft signs as candidate endophenotypes for schizophrenia: A shooting star or a Northern star? Neurosci Biobehav Rev, 2008, 32: 951-957

10 Allen A J, Griss M E, Folley B S, et al. Endophenotypes in schizo- 
phrenia: A selective review. Schizophr Res, 2009, 109: 24-37

11 Eack S M, Merman D E, Montrose D M, et al. Social cognition deficits among individuals at familial high risk for schizophrenia. Schizophr Bull, 2010, 36: 1081-1088

12 Braff D L, Freedman R. The importance of endophenotypes in studies of the genetics of schizophrenia. In: Davis K L, Charney D, Coyle J T, et al., eds. Neuropsychopharmacology: The Fifth Generation of Progress. Philadelphia: Lippincott Williams \& Wilkins, 2002. 703716

13 Braff D L, Freedman R, Shork N J, et al. Deconstructing schizophrenia: An overview of the use of endophenotypes in order to understand a complex disorder. Schizophr Bull, 2007, 33: 21-32

14 Greenwood T A, Braff D L, Cadenhead K S, et al. The Consortium on the Genetics of Schizophrenia (COGS): Preliminary heritability analyses of endophenotypic measures for schizophrenia. Arch Gen Psychiatry, 2007, 64: 1242-1250

15 Husted J A, Lim S, Chow E W, et al. Heritability of neurocognitive traits in familial schizophrenia. Am J Med Gen (Neuropsychiatric Gen), 2009, 150B: 845-853

16 Faraone S V, Seidman L J, Kremen W S, et al. Structural brain abnormalities among relatives of patients with schizophrenia: Implications for linkage studies. Schizophr Res, 2003, 60: 125-140

17 Seidman L J, Wencel H E. Genetically mediated brain abnormalities in schizophrenia. Curr Psychiatry Rep, 2003, 5: 135-144

18 Gottesman I I, Shields J. Schizophrenia and Genetics: A Twin Study Vantage Point. New York: Academic Press, 1972

19 Tsuang M T. Genetics, epidemiology and the search for the causes of schizophrenia. Am J Psychiatry, 1994, 151: 3-6

20 Faraone S V, Kremen W S, Lyons M J, et al. Diagnostic accuracy and linkage analysis: How useful are schizophrenia spectrum phenotypes? Am J Psychiatry, 1995, 152: 1286-1290

21 Faraone S V, Seidman L J, Kremen W S, et al. Neuropsychological functioning among the nonpsychotic relatives of schizophrenic patients: A diagnostic efficiency analysis. J Abnormal Psychol, 1995, 104: 286-304

22 Woodberry K A, Giuliano A J, Seidman L J. Premorbid IQ in schizophrenia: A meta-analytic review. Am J Psychiatry, 2008, 165: 579-587

23 Mesholam-Gately R, Giuliano A J, Faraone S V, et al. Neurocognition in first-episode schizophrenia: A meta-analytic review. Neuropsychologia, 2009, 23: 315--336

24 Heinrichs R W, Zakzanis K K. Neurocognitive deficit in schizophrenia: A quantitative review of the evidence. Neuropsychologia, 1998, 12: 426-445

25 Gur R E, Nimgaonkar V L, Almasy L, et al. Neurocognitive endophenotypes in a multiplex multidimensional family study of schizophrenia. Am J Psychiatry, 2007, 164: 813-819

26 Green M F. What are the functional consequences of neurocognitive deficits in schizophrenia? Am J Psychiatry, 1996, 153: 321-330

27 Green M F, Kern R S, Braff D L, et al. Neurocognitive deficits and functional outcome in schizophrenia: Are we measuring the "right stuff'? Schizophr Bull, 2000, 26: 119-136

28 Goldberg T E, David A, Gold J M. Neurocognitive impairments in schizophrenia: Their character and role in symptom formation. In: Weinberger D R, Harrison P J, eds. Schizophrenia. Oxford: Wiley-Blackwell, 2011. 142-162

29 Nuechterlein K H, Green M F, Kern R S, et al. The MATRICS consensus cognitive battery. Part 1: Test selection, reliability, and validity. Am J Psychiatry, 2008, 165: 203-213

30 Kern R S, Nuechterlein K H, Green M F, et al. The MATRICS Consensus cognitive battery. Part 2: Co-norming and standardization. Am J Psychiatry, 2008, 165: 214-220

31 Kern R S, Green M F, Nuechterlein K H, et al. NIMH-MATRICS survey on assessment of neurocognition in schizophrenia. Schizophr Res, 2004, 72: 11-19

32 Dickinson D, Iannone V N, Wilk C M, et al. General and specific cognitive deficits in schizophrenia. Biol Psychiatry, 2004, 55: 826-833

33 Dickinson D, Ragland J D, Gold J M, et al. General and specific cog- nitive deficits in schizophrenia: Goliath defeats David? Biol Psychiatry, 2008, 64: 823-827

34 Dickinson D, Ragland J D, Calkins M E, et al. A comparison of cognitive structure in schizophrenia patients and healthy controls using confirmatory factor analysis. Schizophr Res, 2006, 85: 20-29

35 Wang Q, Vassos E, Deng W, et al. Factor structures of the neurocognitive assessments and familial analysis in first-episode schizophrenia patients, their relatives and controls. Aust N Z J Psychiatry, 2010, 44: 109-119

36 Nuechterlein K H, Barch D M, Gold J M, et al. Identification of separable cognitive factors in schizophrenia. Schizophr Res, 2004, 72: 29-39

37 Binder L M, Iverson G L, Brooks B L. To err is human: "Abnormal" neuropsychological scores and variability are common in healthy adults. Arch Clin Neuropsychol, 2009, 24: 31-46

38 Wechsler D. WAIS-III WMS-III Technical Manual. San Antonio: The Psychological Corporation: Harcourt Brace and Company, 1997

39 Squire L R, Shimamura A. The neuropsychology of memory dysfunction and its assessment. In: Grant I, Adams K M, eds. Neuropsychological Assessment of Neuropsychiatric Disorders. Oxford: Oxford University Press, 1996. 232-262

40 Fitch K, Bernstein S J, Aguilar M D, et al. The RAND/UCLA Appropriateness Method User's Manual. Santa Monica, California: RAND, 2001

41 Nuechterlein K H, Green M F. MATRICS Consensus Cognitive Battery. Los Angeles: MATRICS Assessment, Inc, 2006

42 Kern R S, Gold J M, Dickinson D, et al. The MCCB impairment profile for schizophrenia outpatients: Results from the MATRICS psychometric and standardization study. Schizophr Res, 2011, 126: 124-131

43 Harvey P D, Green M F, Nuechterlein K H. Latest developments in the MATRICS process. Psychiatry, 2010, 7: 49-52

44 Keefe R S, Fox K H, Harvey P D, et al. Characteristics of the MATRICS consensus cognitive battery in a 29-site antipsychotic schizophrenia clinical trial. Schizophr Res, 2011, 125: 161-168

45 Buchanan R W, Davis M, Goff D, et al. A summary of the FDANIMH-MATRICS workshop on clinical trial design for neurocognitive drugs for schizophrenia. Schizophr Bull, 2005, 31: 5-19

46 Green M F, Nuechterlein K H, Kern R S, et al. Functional co-primary measures for clinical trials in schizophrenia: Results from the MATRICS psychometric and standardization study. Am J Psychiatry, 2008, 165: 221-228

47 Green M F, Schooler N R, Kern R S, et al. Evaluation of co-primary measures for clinical trials of cognition enhancement in schizophrenia. Am J Psychiatry, 2011, 168: 400-407

48 Silverstein S M, Jaeger J, Donovan-Lepore A M, et al. A comparative study of the MATRICS and IntegNeuro cognitive assessment batteries. J Clin Exp Neuropsychol, 2010, 32: 937-952

49 Barnett J H, Robbins T W, Leeson V C, et al. Assessing cognitive function in clinical trials of schizophrenia. Neurosci Biobehav Rev, 2010, 34: 1161-1177

50 Bleuler E. Dementia Praecox or the Group of Schizophrenias. New York: International Universities Press, 1911/1950

51 Gottesman I I. Schizophrenia Genesis: The Origin of Madness. New York: Freeman, 1991

52 Tsuang M T, Stone W S, Faraone S V. Schizophrenia: A review of genetic studies. Harv Rev Psychiatry, 1999, 7: 185-207

53 Glatt S J, Faraone S V, Tsuang M T. Genetic risk factors for mental disorders: General principles and state of the science. In: Tsuang M T, Stone W S, Lyons M J, eds. Recognition and Prevention of Major Mental and Substance Use Disorders. Washington D.C.: American Psychiatric Publishing, Inc, 2007. 3-20

54 Kendler K S. Twin studies of psychiatric illness: An update. Arch Gen Psychiatry, 2001, 58: 1005-1014

55 Rado S. Dynamics and clssification of disordered behavior. Am J Psychiatry, 1953, 110: 406-416

56 Rado S. Theory and therapy: The theory of schizotypal organization and its application to the treatment of decompensated schizotypal behavior. In: Scher S C, Davis H R, eds. The Outpatient Treatment of 
Schizophrenia. New York: Grune \& Stratton, 1960. 87-101

57 Meehl P E. Schizotaxia, schizotypy, schizophrenia. Am Psychol, 1962, 17: 827-838

58 Meehl P E. Schizotaxia revisited. Arch Gen Psychiatry, 1989, 46: 935-944

59 Faraone S V, Green A I, Seidman L J, et al. "Schizotaxia": Clinical implications and new directions for research. Schizophr Bull, 2001, 27: $1-18$

60 Thaker G K. Endophenotypic studies in schizophrenia: Promises and challenges. Schizophr Bull, 2007, 33: 1-2

61 Stone W S, Faraone S V, Seidman L J, et al. Searching for the liability to schizophrenia: Concepts and methods underlying genetic high-risk studies of adolescents. J Child Adolesc Psychopharmacol, 2005, 15: 403-417

62 Tsuang M T, Stone W S, Auster T L. Prevention of schizophrenia. Expert Rev Neurotherapeutics, 2010, 10: 1165-1174

63 Dazzan P, Kravariti E, Fearon P, et al. Is the development of schizophrenia predictable? In: Stone W S, Faraone S V, Tsuang M T, eds. Early Clinical Intervention and Prevention in Schizophrenia. Totowa, New Jersey: Humana Press, 2004. 225-252

64 Cannon T D, Cadenhead K S, Cornblatt B, et al. Prediction of psychosis in youth at high clinical risk: A multisite longitudinal study in North America. Arch Gen Psychiatry, 2008, 65: 28-37

65 Simon, A E, Roth B, Zmilacher S, et al. Developing services for the early detection of psychosis: A critical consideration of the current state of the art. Eur Child Adolesc Psychiatry, 2007, 16: 96-103

66 Simon A E, Umbricht D. High remission rates from an initial ultra-high risk state for psychosis. Schizophr Res, 2010, 116: 168-172

67 Erlenmeyer-Kimling L. Early neurobehavioral deficits as phenotypic indicators of the schizophrenia genotype and predictors of later psychosis. Am J Med Genet (Neuropsychiatric Genet), 2001, 105: 23-24

68 Mortensen P B, Pedersen M G, Pedersen C B. Psychiatric family history and schizophrenia risk in Denmark: Which mental disorders are relevant? Psychol Med, 2010, 40: 201-210

69 Tsuang M T, Stone W S, Seidman L J, et al. Treatment of nonpsychotic relatives of patients with schizophrenia: Four case studies. Biol Psychiatry, 1999, 41: 1412-1418

70 Tsuang M T, Gilbertson M W, Faraone S V. Genetic transmission of negative and positive symptoms in the biological relatives of schizophrenics. In: Marneros A, Tsuang M T, Andreasen N, eds. Positive vs. Negative Schizophrenia. New York: Springer-Verlag, 1991. 265-291

71 Tsuang M T, Stone W S, Tarbox S I, et al. An integration of schizophrenia with schizotypy: Identification of schizotaxia and implications for research on treatment and prevention. Schizophr Res, 2002, 54: $169-175$

72 Snitz B E, MacDonald III A W, Carter A S. Cognitive deficits in unaffected first-degree relatives of schizophrenia patients: A metaanalytic review of putative endophenotypes. Schizophr Bull, 2006, 32: 179-194

73 Sitskoorn M M, Aleman A, Ebisch S J H, et al. Cognitive deficits in relatives of patients with schizophrenia: A meta-analysis. Schizophr Res, 2004, 71: 285-295

74 Kremen W S, Seidman L J, Pepple J R, et al. Neuropsychological risk indicators for schizophrenia: A review of family studies. Schizophr Bull, 1994, 20: 103-119

75 Chan R C K, Stone W S, Hsi X. Neurological and neuropsychological endophenotypes in schizophrenia spectrum disorders. In: Ritsner M S, ed. Textbook of Schizophrenia Spectrum Disorders. Volume II: Phenotypic and Endophenotypic Presentation. Heidleberg: Springer, 2011. 325-349

76 Stone W S, Faraone S V, Seidman L J, et al. Concurrent validation of schizotaxia: A pilot study. Biol Psychiatry, 2001, 50: 434-440

77 Weissman M M, Bothwell S. Assessment of social adjustment by patient self-report. Arch Gen Psychiatry, 1976, 33: 1111-1115

78 American Psychiatric Association. Diagnostic and Statistical Manual of Mental Disorders (DSM-IV), 4th ed. Washington DC: American Psychiatric Association, 1994

79 Derogatis L R. Symptom Checklist-90-R (SCL-90-R). Minneapolis, MN: Computer Systems, Inc, 1993

80 Andreasen N C. The Scale for the Assessment of Negative Symptoms (SANS). Iowa City, Iowa: The University of Iowa, 1983

81 Stone W S, Seidman L J. Towards a model of memory enhancement in schizophrenia: Glucose administration and hippocampal function. Schizophr Bull, 2008, 34: 93-108

82 Harvey P D. Pharmacological cognitive enhancement in schizophrenia. Neuropsychol Rev, 2009, 19: 324-335

Open Access This article is distributed under the terms of the Creative Commons Attribution License which permits any use, distribution, and reproduction in any medium, provided the original author(s) and source are credited. 